\title{
Bilateral cochlear implantation: simultaneous versus sequential
}

\author{
Reham Elrashidy ${ }^{1 *}$, Ahmed Mohamed Khater ${ }^{2}$, Mohmad Shabana $^{3}$, Ahmed Khashaba ${ }^{4}$ and Adel Tharwat ${ }^{5}$
}

\begin{abstract}
Background: Bilateral cochlear implantation (BiCl) has been provided with an intent to promote binaural hearing and improve quality of life (QoL). Binaural hearing maximum benefit could be optimized with $\mathrm{BiCl}$, when indicated in the early stages of life through preserving the cortical hearing development. Actually, BiCl benefits wide variables among patients, and in this work, we aimed to analyze the benefit of bilateral cochlear implantation over unilateral and the simultaneous over sequential implantation in peri- and post-lingual patients. The analysis based on common clinically used audiological tests such as aided hearing threshold and speech perception measures.

Results: The results of the aided threshold and speech perception measures in all patients revealed a significantly better response in the bilateral implantations than in unilateral each implant separately. Overall, the best response was seen in the simultaneous bilateral group followed by sequential bilateral groups. In post-lingual patients, a better response was observed in sequential and similar bilateral than in sequential and different device groups with significant differences.

Change of performance across time revealed aided threshold improvement of the from 3 to 6 months. In speech perception measures, the post-lingual group revealed significant improvement of high-context sentence test (HCST) scores from 3 to 6 months and 12 months. In the peri-lingual group, Early Speech Perception test (ESPT) revealed a change in performance with time at 3,6, and 12 months. In addition, the simultaneous patient's scores were significantly better than sequential similar patient's scores.

Conclusion: The main finding of our study was that bilateral cochlear implants (BiCl) are better than unilateral cochlear implant, and simultaneous $\mathrm{BiCl}$ is preferred than the sequential cochlear implant even after escaping the early stage of language development.
\end{abstract}

Keywords: Bilateral cochlear implantation, Simultaneous cochlear implant, Sequential cochlear implant

\section{Background}

Bilateral cochlear implantation (BiCI) is considered as an accepted medical practice in clinically appropriate adults and children [1]. Among some groups, it is considered the standard of care for children with bilateral profound sensorineural hearing loss [2]. The objective benefits of $\mathrm{BiCI}$ correspond with the primary benefits of bilateral hearing, which include improved speech perception in noise and localization abilities [3].

\footnotetext{
*Correspondence: rehamelrashedy@yahoo.com; raelrashidy@dha.gov.ae ${ }^{1}$ Hearing and Balance Clinic, ENT Department, Dubai Hospital,DHA, Abu Bakr Al Siddiq Street, Dubai 7272, United Arab Emirates

Full list of author information is available at the end of the article
}

Outcome measures suggest that these benefits of sound localization and hearing better in noisy environments, implanting the better ear aiming for maximizing success from the implant and quality of life, are present in the majority of recipients, although their performance is still not at the level of normal-hearing listeners with true binaural capabilities. Other improvements that have not been systematically measured, but have the potential to be measured include facilitation of language acquisition, learning, cognition, and memory, the fact that the "better ear" is guaranteed to be implanted for the better quality of life [4]. The benefits of BiCI measures wide variables among patients. To ensure the maximal 
Table 1 Patients' descriptive data

\begin{tabular}{|c|c|c|c|}
\hline & Category & Frequency & Percentage \\
\hline \multirow[t]{2}{*}{ Gender } & Female & 7 & 46.7 \\
\hline & Male & 8 & 53.3 \\
\hline \multirow[t]{2}{*}{ The onset of hearing loss } & Postlingual & 8 & 53.3 \\
\hline & Perilingual & 7 & 46.7 \\
\hline \multirow[t]{7}{*}{ Type of the speech processor } & Opus2 & 4 & 26.7 \\
\hline & Neurelec digisonic and Sonata & 1 & 6.7 \\
\hline & Neurelec digisonic and Freedom & 1 & 6.7 \\
\hline & Sonata & 3 & 20 \\
\hline & Harmony & 4 & 26.7 \\
\hline & Freedom & 1 & 6.7 \\
\hline & N5 & 1 & 6.7 \\
\hline
\end{tabular}

potential for binaural processing, the reported age with maximum potential advantages of early plasticity and optimum binaural benefit is during the first 3.5 years of life [5].

The determination of which ear to implant can be influenced by several factors. Many clinics routinely implant the ear with the least amount of residual hearing, while other clinics routinely implant the patient's "best" hearing ear. Some clinics determine the ear of the implant on a case-by-case basis, while others leave this decision up to the patient and/or the parents. Recently, many clinics have begun to offer bilateral implants, eliminating the need to decide about which ear to implant (simultaneous bilateral implantation) or (sequential bilateral implantation) that takes place weeks, months, or even years apart [6].

Simultaneous BiCI promotes the development of bilateral auditory pathway in tandem and is less resourceconsuming for rehabilitation compared with sequential bilateral implantation in children [7].

\section{Methods}

\section{Patients}

This study was done at ENT Medical Center for a cochlear implant in Riyadh Saudi Arabia, in the period from January 2012 to January 2017. Patients enrolled in the study included patients with bilateral profound hearing loss, and their age ranged between 5 and 45 years old. All selected patients acquired language with language age above 3 years, ability to repeat, good visual attention, and average mental ability. The study included 15 patients ( 7 females and 8 males), and bilateral implanted patients show a heridofamilial hearing loss as the most common cause of hearing loss, 8 post-lingual and 7 perilingual cases. They did not have any additional disabilities. All had been full-time cochlear implant (CI) users. As regards the used speech processors, only in 2 cases we used 2 different devices; MXM (Nurelec digisonic) with MEDEL (Sonata) for the first patient and MXM (Neurelec digisonic) with Cochlear (freedom) for the other one. The left 13 cases with bilateral similar implants; 4 cases with bilateral MEDEL (Opus2), 3 cases with bilateral MEDEL (Sonata), 2 cases with bilateral cochlear (1 bilateral freedom and 1 bilateral system 5 ), and 4 cases with bilateral Advanced Bionics (AB) (Harmony with 1 J, Table 1 ).

As regards the type of surgery, 9 patients were implanted sequentially with a large gap duration range between 8 months and 2 years. On the other hand, 6 patients were implanted simultaneously. All maps were fitted individually to achieve the best possible hearing sensation for each patient (Table 2).

\section{Equipment}

Laptop with specific fitting software for each device, the HiRes clinical fitting tool, version 1.6.8 for Advanced Bionics device (AB); Digimap SP version 3.0 for Neurelec device (MXM); Custom sound version 3.1 for Freedom and Nucleus system 5; and finally, MAESTRO system version 4.0 for MEDEL are the equipment used in the

Table 2 Patients' descriptive data

\begin{tabular}{llll}
\hline & Cochlear implant speech processors & Frequency & Percentage \\
\hline Type of implant & Similar implants & 13 & 53.3 \\
& Different implants & 2 & 46.7 \\
Type of surgery & Sequential implantation (7 of similar implants + 2 of different implants) & 9 & 86.7 \\
& Simultaneous implantation (6 of similar implants) & 6 & 13.3 \\
\hline
\end{tabular}


study. In addition, each patient's own speech processor; pure tone audiometer, two-channel clinical audiometer, AC 40 audiometer (InteracousticsA/S, Denmark). The audiometer is equipped with a sound-field connection. Double-wall sound-treated booth $3.5 \times 3 \mathrm{~m}$.

\section{Mapping}

After a sufficient healing period, initial programming and activation of both devices occurred on the same day for simultaneously implanted devices. In sequentially implanted devices, each device activated independently to the other. Each ear underwent independent mapping and each device mapped using its specific software at the time of the study.

Determination of cochlear implant stimulation levels and other adjustable parameters are similar to the practices used for unilateral recipients, in addition to bilateral match to the adjustable parameters, such as stimulation rate, input dynamic range, and frequency allocation.

In $\mathrm{BiCI}$ programming, adjustments were made to achieve equal loudness for each frequency. However, there is no agreed way to determine the most effective strategy to optimize bilateral stimulation levels. In our practice, we program the initial cochlear implant while the other one is switched off., and then, we do the second map in reference to the first one. In most cases, bilateral summation addressed by providing a small global decrease in the upper stimulation levels relative to the unilateral conditions.

\section{Aided threshold}

The patient seated in a double-wall sound-treated booth $1 \mathrm{~m}$ away from $45{ }^{\circ} \mathrm{C}$ azimuth to the back loudspeaker in sound field condition. Warble tone at $500 \mathrm{~Hz}, 1000$ $\mathrm{Hz}, 2000 \mathrm{~Hz}$, and $4000 \mathrm{~Hz}$ were delivered through a loudspeaker. Aided sound-field thresholds were measured. Instructions were given to the patients to raise the hand (or pressing a button) when hearing the stimulus even though it may be very faint and barely audible. To minimize the impact of the fluctuation in the input, the stimuli were presented in an ascending manner in 5$\mathrm{dB}$ steps. A stimulus with about 1-2 $\mathrm{s}$ in duration was used (sufficient duration to activate the internal circuits of the implant). The interval between stimulus presentations was $30 \mathrm{~s}$ (sufficient time for the duration of the release time before presenting the next stimulus). The aided threshold measured when both devices were activated and re-measured again when only each device, separately. In order to compare performance change with time of aided threshold, the test repeated at 3,6 , and 12 months of the second implant.

High-context speech test (HCST) applied in the postlingual group of patients (8 cases). HCST is an open-set speech recognition Arabic tests developed by Abdel Maksoud et al. in 1990 [8]. HCST materials composed of fifty highly predictable sentences; in this study, we used a subset of "ten sentences" in each testing condition. Firstly, HCST is quietly done when the patient was using one implant while the other implant is off. This procedure repeated with each implant, then with both implants switched on, i.e., bilateral versus unilateral. The speech material was presented at the MCL of each patient.

Every time, we used a different list of ten sentences. In scoring, we used the way for the whole sentence meaning; one point was given for a sentence if all keywords are correct. HCST is quite repeated at 3,6 , and 12 months of the second implant to assess the change of performance with time.

Secondly, HCST has done in noise 1 year after the second implantation bilateral versus unilateral as before to study the binaural summation benefit in both quiet and noise. Speech materials routed through at fixed intensity level $70 \mathrm{dBhl}$ through the loudspeakers. The level could be changed to MCL of each patient; the test is repeated in varied speech noise in three sentences at SNR $0 \mathrm{~dB}$, and if the correct keyword score was less than $50 \%$, the test is repeated by decreasing the noise at $+10 \mathrm{~dB}$ SNR up to $+15 \mathrm{~dB}$ SNR maximum. After the optimization of SNR for each patient, the HCST in noise is carried out.

The standard Early Speech Perception (ESP) battery is applied to the peri-lingual group ( 7 cases) as this test is recommended for children with language age above 3 years. The ESP battery consists of a pattern perception (pp) subtest and two-word identification subtests including spondee, trochee identification (TI), and monosyllable identification (MI) subtests. The test was developed by El-Kholy in 2000 [9]. In the pp, 12 words in four different durational or stress patterns are presented. These are three monosyllabic words, three iamb words (bisyllabic words with stress on the second syllable), three trochee words (bisyllabic words with stress on the first syllable), and three trisyllabic words. The TI subset is composed of 12 trochee words that widely varying vowels and consonants. The monosyllabic identification subset (MI) consists of twelve monosyllabic words with a different middle vowel sound. Pictures of the recorded material were formulated into three colored plates representing the 3 subsets, each composed of 12 pictures. The child allowed to some practice item in an informal situation outside the sound-treated room using both visual and auditory input, the child was allowed to wear the CI adjusted at the MCL. The child was given the colored plate for the test/subset and was instructed to point to the word he heard. There are two methods of scoring in PP subset, pattern correct 
Table 3 Aided threshold 6 months after bilateral implantation

\begin{tabular}{|c|c|c|c|c|}
\hline & Frequency tested $(\mathrm{Hz})$ & First implant & Second implant & Bilateral implant \\
\hline \multirow[t]{4}{*}{ Simultaneous } & 500 & 30 & 35 & 20 \\
\hline & 1000 & 30 & 30 & 15 \\
\hline & 2000 & 25 & 30 & 15 \\
\hline & 4000 & 30 & 35 & 20 \\
\hline \multirow[t]{4}{*}{ Sequential and similar } & 500 & 30 & 35 & 20 \\
\hline & 1000 & 25 & 35 & 15 \\
\hline & 2000 & 25 & 40 & 20 \\
\hline & 4000 & 30 & 40 & 25 \\
\hline \multirow[t]{4}{*}{ Sequential and different } & 500 & 35 & 35 & 25 \\
\hline & 1000 & 30 & 35 & 20 \\
\hline & 2000 & 30 & 30 & 25 \\
\hline & 4000 & 35 & 35 & 25 \\
\hline
\end{tabular}

or word correct; we used pattern correct in our study as identification of temporal pattern was evaluated. Since each word was repeated twice in different randomization, a total number of 24 words were administrated in this subset. The chance level for PP pattern score was 6 . While the trochee identification subset scored for word correct with a chance level 2 out of 24 words. The same applied to the monosyllabic identification subset. In our study, the test was done in the peri-lingual seven cases. First, the patient was using one implant while the other implant is off in each implant separately and then repeated with both implants switched on. Then, the test repeated to assess the change of performance with time at 3,6 , and 12 months of the second implant.

\section{Results}

\section{Aided threshold}

The patients were divided according to the surgery and the processor used in the study into three groups: the first was the simultaneous implantation group, the second is sequential with similar implants group (sequential and similar), and the third was sequential with different implants group (sequential and different). The results showed an improvement of aided threshold across the four audiometric frequencies 6 months after the second implantation. A better response was observed in the bilateral implant than in unilateral each implant separately in all patients (Table 3). There are significant differences among the three groups; the bilateral implant is found to be more favorable than unilateral each implant separately

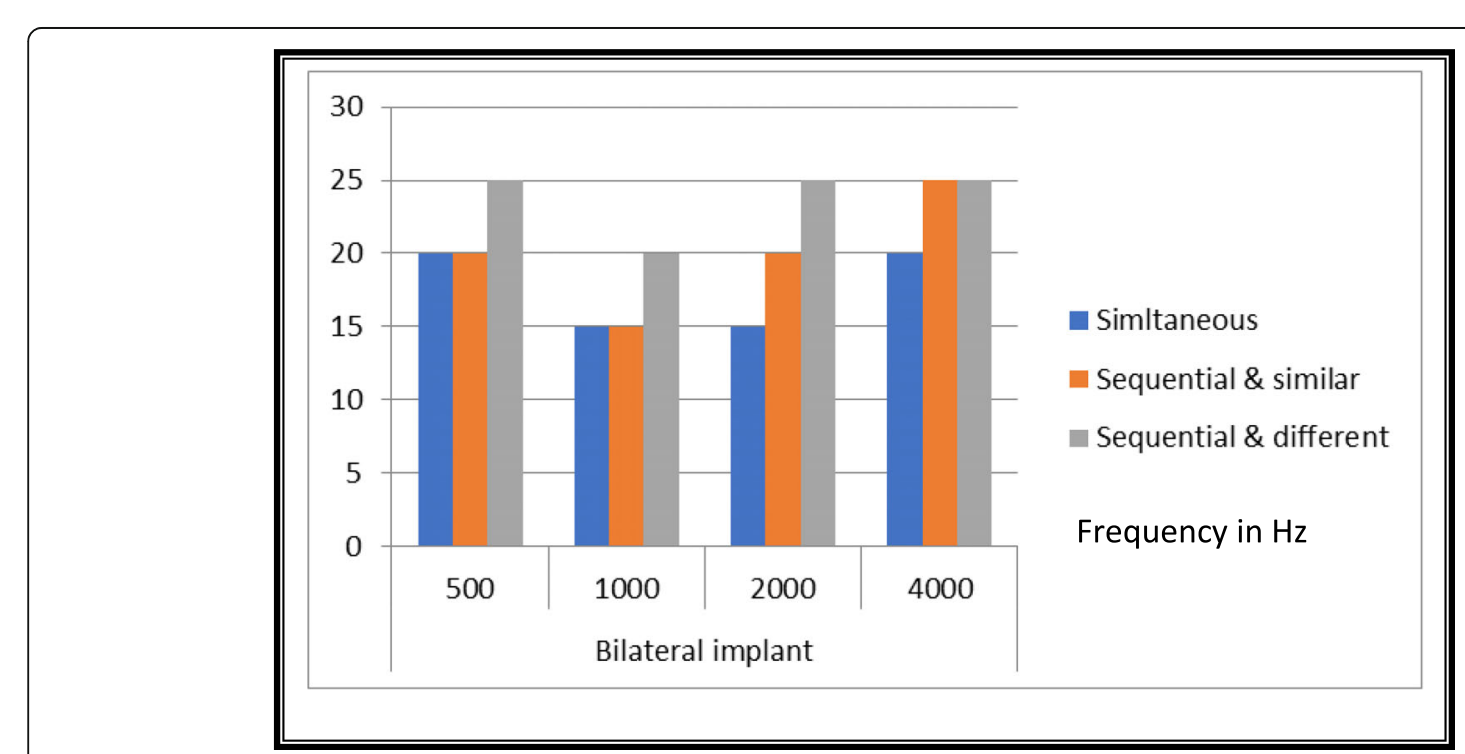

Fig. 1 Comparison of the aided threshold with simultaneous versus sequential similar and different bilateral implants 6 months after the second implantation 


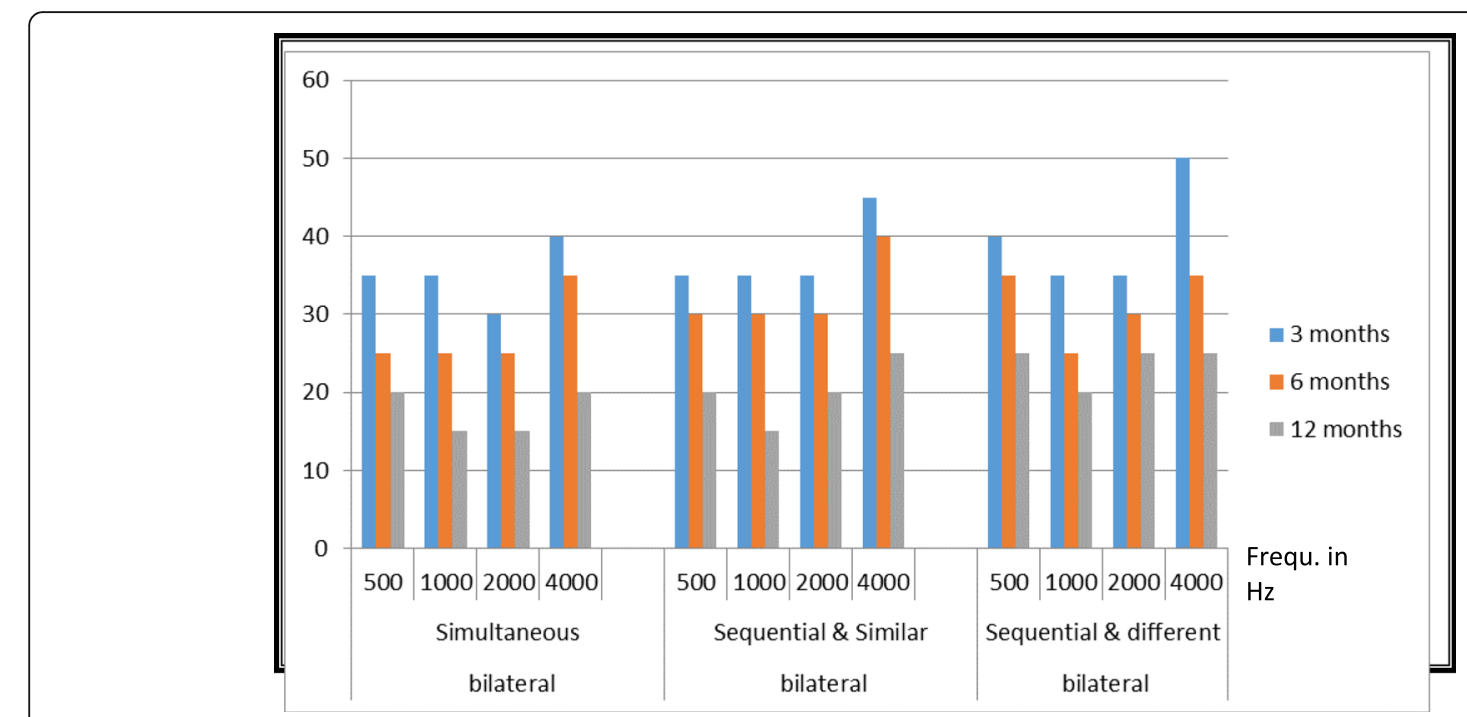

Fig. 2 Comparison of aided threshold performance change of bilateral implant at 3, 6, and 12 months of the second implant

$(P<0.05)$. In addition, the best-aided response was seen in the simultaneous bilateral group followed by sequential bilateral groups and showed better-aided response in sequential and similar bilateral than in sequential and different devices group (Fig. 1). The statistical paired $t$ test analysis revealed that there is a significant difference $(P=$ $.023<0.05)$ between the simultaneous bilateral group and sequential device groups.

\section{Change of performance in aided threshold with time at 3, 6 , and 12 months}

The averages of the aided threshold between patients with simultaneous, sequential similar, and sequential different bilateral implants collected at 3, 6, and 12 months of the second implants (change of performance/time) across the four audiometric frequencies 500, 1000, 2000, and 4000 $\mathrm{Hz}$ (Fig. 2). The results showed an improvement in the aided threshold from 3, 6, and 12 months. The statistical

Table 4 HCST for the post-lingual group, comparison of HCST with the bilateral and unilateral implant for post-lingual after 1 year of the second implantation

\begin{tabular}{llll}
\hline Patient & Bilateral implant (\%) & Best implant (\%) & Second implant (\%) \\
\hline P1 & 60 & 60 & 50 \\
P2 & 60 & 50 & 30 \\
P3 & 60 & 50 & 40 \\
P4 & 60 & 50 & 40 \\
P5 & 70 & 60 & 30 \\
P8 & 80 & 70 & 60 \\
P11 & 80 & 70 & 60 \\
P12 & 70 & 60 & 50 \\
\hline
\end{tabular}

paired sample test views the significant difference in aided response between each two periods $(P=.000<0.05)$.

\section{Arabic speech perception tests \\ High-context sentence test (HCST)}

HCST was done for the 8 post-lingual patients. The results collected when bilateral implants switched on and with each unilateral implant separately 1 year after the second implantation. The results showed a gradual improvement in HSCT scores with the best score at bilateral conditions (Table 4). The statistical paired $t$ test indicates the existence of significant differences in bilateral versus each unilateral implant $(P=.001<0.05)$.

Simultaneous versus sequential bilateral implants In the post-lingual 8 patients, HCST average scores were collected: the simultaneous bilateral implants (3 cases; P8, P11, and P12), sequential and similar bilateral implants (3 cases; P1, P4, and P5), and sequential and different bilateral implants (2 cases, P2 and P3) 1 year after the second implant. The highest HCST score was observed in the simultaneously implanted group, followed by sequential and similar bilateral implants with the least score in sequential and different implanted group (Table 5). The statistical analysis showed that there is a significant

Table 5 HCST average scores with bilateral implants 1 year after the second implant showed the best score in the simultaneously implanted group followed by sequentially and similar implanted group with least score in sequential and different one

\begin{tabular}{llll}
\hline & \multicolumn{3}{l}{ Bilateral implantation } \\
\cline { 2 - 4 } & Simultaneous & Sequential and similar & Sequential and different \\
\hline HCST & $77 \%$ & $63 \%$ & $60 \%$ \\
\hline
\end{tabular}




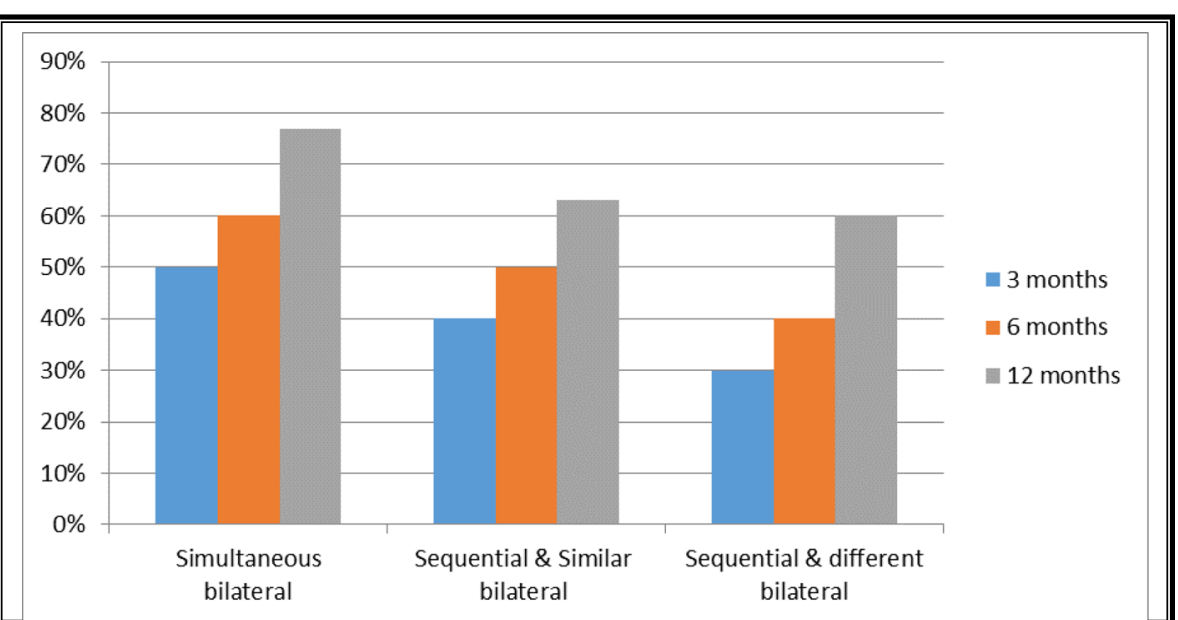

Fig. 3 Comparison of HCST at 3, 6, and 12 months for post-lingual group

difference $(P=.025<0.05)$ between the simultaneous bilateral group and sequential and similar device group. Furthermore, a significant difference $(P=.017<0.05)$ between the simultaneous bilateral group and sequential and different device group.

\section{Change of HCST performance with time at 3, 6, and} 12 months HCST average scores for simultaneous, sequential and similar, and sequential and different bilateral implants at 3, 6, and 12 months of the second implants (change of performance/time) are shown in (Fig. 3). Visualizing the comparison among groups showed that there is a gradual improvement of HCST scores from 3 to 6 months and from 6 to 12 months, respectively. In addition, the high scores were observed in the simultaneous bilateral group than in the sequential and similar bilateral groups with the least scores in sequential and different bilateral implants. The statistical paired $t$ test views the significant difference in HCST scores between each two periods.

\section{Peri-lingual patients tested with Early Speech Perception test (ESP)}

This subsection is devoted to ESP scores of bilateral implant and unilateral implants with a change in performance with time at 3, 6, and 12 months for perilingual group. The group is divided into simultaneous bilateral implant group (3 cases), and sequential and similar bilateral implant group (4 cases) (Table 6).

We observed changes of improvement in the performance with time, and the best scores were collected at 12 months after the second implantation. It is also observed that simultaneous patient's scores are better than sequential and similar patient's scores (Figs $4 a-c)$. One year after the second implant statistical paired $t$ test views, there is a significant difference $(P=.003<0.05)$ between the scores in a bilateral implant and unilateral (best) implant. Furthermore, a significant difference $(P=.001<0.05)$ between the simultaneous, and sequential and similar in bilateral versus unilateral implant.

\section{Effect of binaural summation using HCST in the post- lingual group in quiet and competing noise}

In a high-context sentence test in quiet and in noise, unilateral versus bilateral scores in the post-lingual group 1 year after the second implant shows an improvement of HCST scores in bilateral CIs better than each unilateral implant. In addition, it has an overall

Table 6 Average scores of ESP test for peri-lingual group

\begin{tabular}{|c|c|c|c|c|c|c|c|c|c|c|}
\hline & & \multicolumn{3}{|c|}{3 months } & \multicolumn{3}{|c|}{6 months } & \multicolumn{3}{|c|}{12 months } \\
\hline & & $\mathrm{PP}$ & $\mathrm{TI}$ & MSI & $\mathrm{PP}$ & $\mathrm{TI}$ & MSI & $\mathrm{PP}$ & $\mathrm{TI}$ & MSI \\
\hline \multirow[t]{2}{*}{ Bilateral implant } & Simultaneous & 63.1 & 20.2 & 4 & 64.3 & 40.5 & 19.4 & 64 & 44.5 & 21.7 \\
\hline & Sequential and similar & 56.1 & 19 & 0 & 60 & 32 & 16.3 & 62 & 40 & 18 \\
\hline \multirow[t]{2}{*}{ Best implant } & Simultaneous & 52.7 & 16.2 & 2.3 & 54.5 & 29.4 & 14.7 & 57.2 & 34.4 & 16.5 \\
\hline & Sequential and similar & 49 & 12 & 0 & 46 & 27 & 10.2 & 47 & 29 & 13 \\
\hline \multirow[t]{2}{*}{ Second implant } & Simultaneous & 36.4 & 8.8 & 1.6 & 44.3 & 26.2 & 8.8 & 46.6 & 28.2 & 12.3 \\
\hline & Sequential and similar & 32 & 6 & 0 & 23.3 & 17 & 6 & 32 & 20 & 11 \\
\hline
\end{tabular}




\section{a: EPS for bilateral implant}

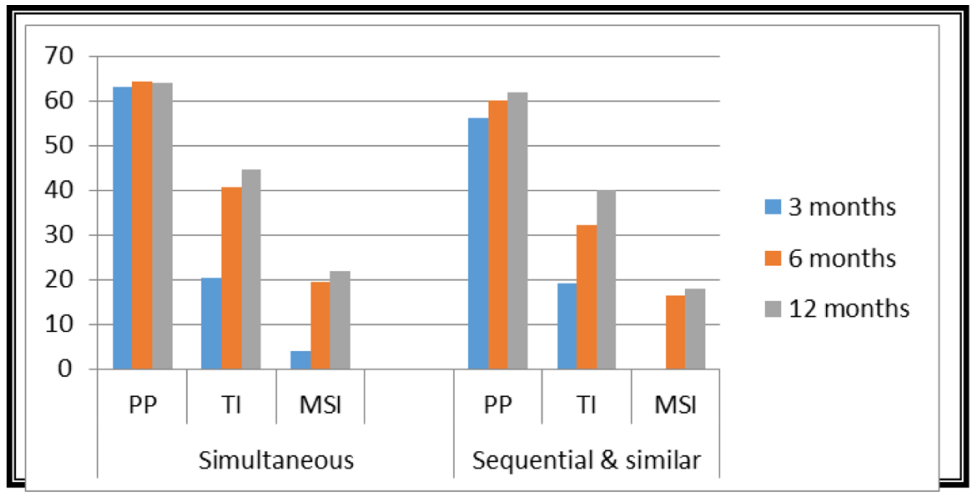

b: EPS for the best implant

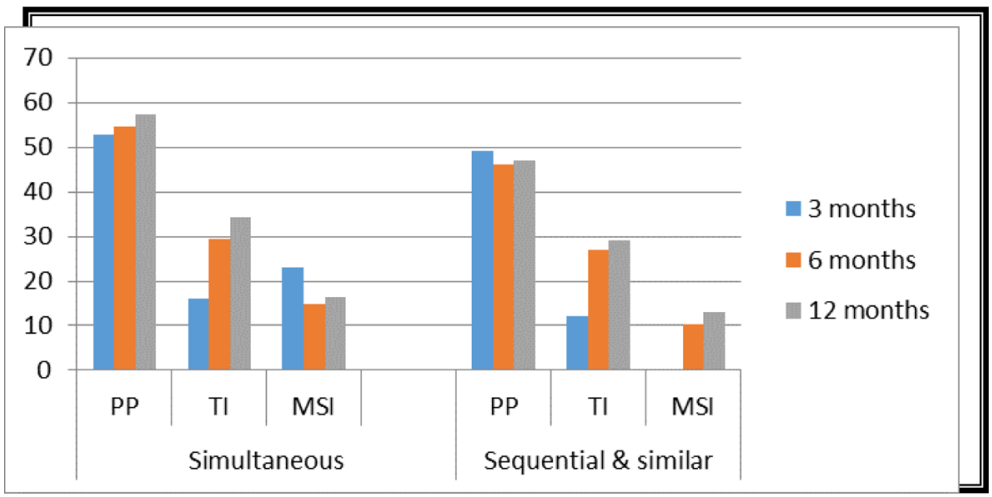

c: EPS for the second implant.

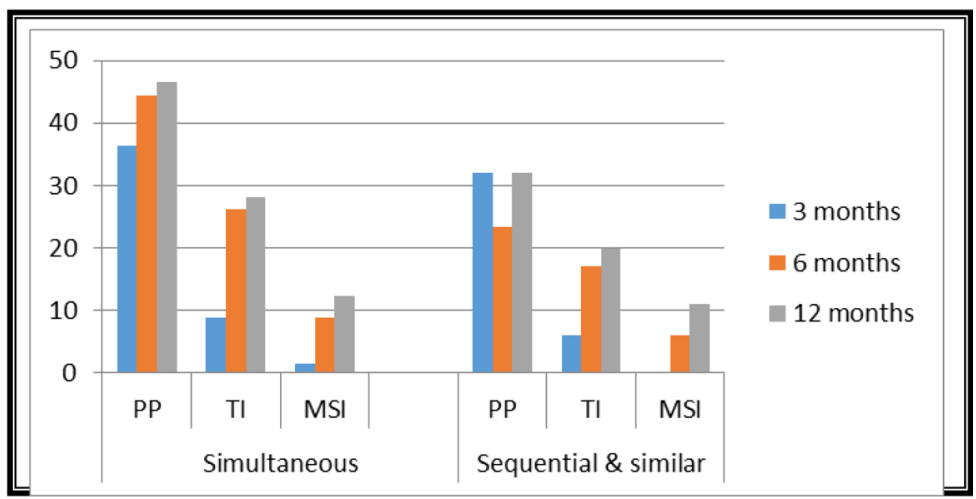

Fig. 4 a EPS for bilateral implant. b EPS for the best implant. $\mathbf{c}$ EPS for the second implant

better performance in quiet than in noise (Table 7). The statistical paired $t$ test views the significant difference when bilateral implants are switched on $(P=.000<$ 0.05 ) in quiet versus in noise.

\section{Discussion}

The cochlear implant is the most successful of all neural prostheses developed to date. It is the most effective prosthesis in terms of restoration of function [10].
Several studies have documented the advantages of $\mathrm{BiCI}$ over unilateral $\mathrm{CI}$ supporting our results. BiCI improved speech intelligibility, speech perception in background noise [11, 12], and sound localization in quiet places $[4,12,13]$ and in noisy situations [14].

Simultaneous BiCI promotes the development of bilateral auditory pathways in tandem and is less resource consuming for rehabilitation compared with sequential bilateral implantation in children [7]. It does not yet 
Table 7 HCST in quiet and in noise in post-lingual group 1 year after the second implant

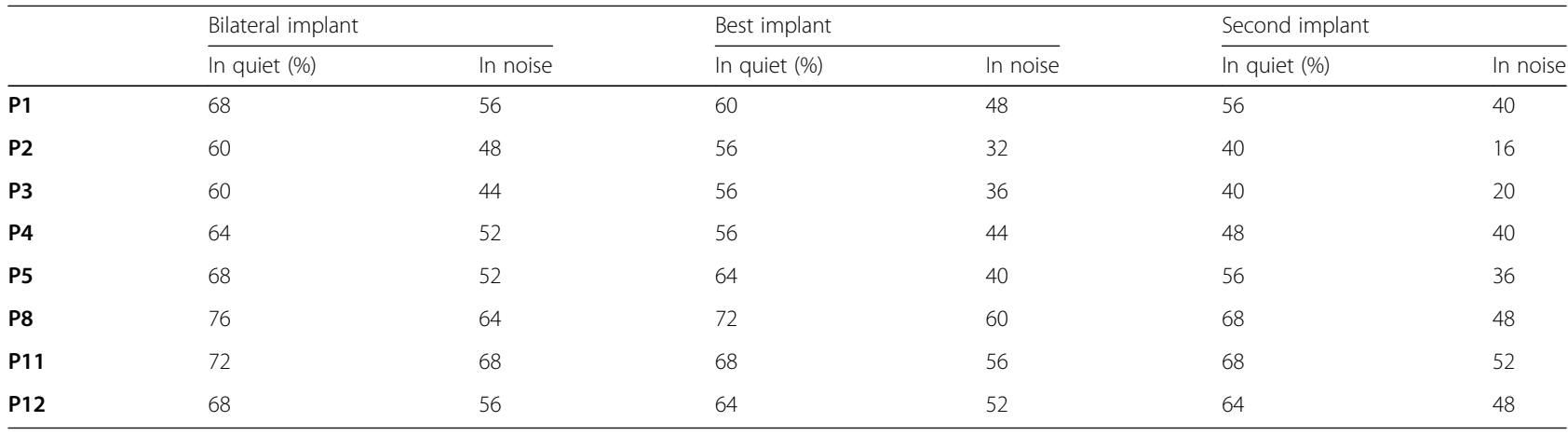

clear whether these asymmetries in sequential $\mathrm{BiCI}$ will persist with longer-term use, but they are not evident in children who receive cochlear implants with short delays of less than 1 year or simultaneously [15]. However, there is marked heterogeneity between studies with respect to the age at the first and second cochlear implant and the optimum delay between the two implants [16]. These studies gave strength to our finding of better speech response in bilateral and simultaneous implanted patients more than those sequentially implanted did. Specifically, all patients in the current study implanted with a long gap duration.

In our study, there was a significant improvement of aided threshold across audiometric frequencies $(0.5,1,2$, and $4 \mathrm{kHz}$ ) in bilateral fitting over unilateral fitting (first and second implant). In addition, we reviewed that aided threshold across audiometric frequencies $(0.5,1,2$, and $4 \mathrm{kHz}$ ) in simultaneous cases was better than the same sequential cases with the least results in different sequential cases with a significant difference.

These results are strongly supported by the research of Baudonck et al. in 2010 who concluded that cochlear implants were the best current alternative for bilateral severe or profound hearing loss, achieving better results in speech perception and development in prelingual children when compared with conventional hearing aids. In addition, Fernandes et al. in 2015 reinforced the benefits of cochlear implants in children with severe or profound hearing loss $[17,18]$.

Our concerned study reviewed the aided response in the same simultaneous patients was better than the same sequential group with the least response in different sequential patients. These results agreed with what Chadha et al. in 2011 reviewed that children with simultaneously implanted $\mathrm{BiCl}$ demonstrated an advantage over children with the sequential implant by using spatial cues to improve speech detection in noise [19].

In our concerned study, speech perception in the post-lingual group tested with HCST was better after bilateral fitting with significant improvement of HCST scores after the second implant than the first and best implants alone. The results agreed with Reiss et al. 2018 who reported a better speech perception in bilateral cochlear implants. They stated that bilateral cochlear implant improves binaural hearing, reduces head shadow effect, and improves speech perception. In addition, the performance of Early Speech Perception test (ESP) in peri-lingual patients over time. The best scores were in bilateral cochlear implant fitting followed by unilateral best implant; the least scores were in the unilateral second implant. Also, there was a gradual improvement of scores from 3 to 6 months and 6 to 12 months after the second implant fitting [20].

Our ESP scores for simultaneous cochlear implant were better than sequential implants (bilateral, unilateral best, and unilateral second implant). The results agreed with what Chadha et al. (2011) concluded that children with simultaneously implanted BiCI demonstrated an advantage over children with a sequential implant with a large gap by using spatial cues to improve speech detection in noise. We have to take into consideration that some patients may be not considered suitable for bilateral implantation. That reasons included developmental delay, residual borderline hearing in the second ear, parental/patient refusal, abnormally poor speech development for age, and abnormal cochlear anatomy precluding implantation. Overall, these findings encourage the use of bilateral cochlear implants over unilateral. However, individual variability among patients [19].

\section{Conclusions}

Optimum bilateral was aided and speech performance was obtained with matching bilateral the same simultaneous and same sequential devices in both peri- and postlingual patients. Least benefits were in different sequential devices in the postlingual patients.

As regards bilateral benefits, "BiCI patient's speech performances were usually better than unilateral CI. Binaural loudness measures revealed that both of tone 
and speech signals were more easily detectable and discriminable in BiCI than unilateral CI conditions.

Accordingly, we are recommending bilateral and simultaneous cochlear implants rather than a unilateral or sequential cochlear implant. However, the wide variability in speech performance among patients could be attributed to different factors, including the areas of hardware and engineering, surgical precision, age of implantation, and pathology of the auditory system in deaf persons. In the future, this will drive our thinking of how we achieve binaural hearing as the targeted way in our work rather than bilateral hearing.

\section{Abbreviations}

AB: Advanced bionic; BiCl: Bilateral cochlear implantation; Cl: Cochlear implant; ESP: Early Speech Perception test; Fig.: Figure; HINT: Hearing in Noise Test; HCST: High-context sentence test; MCL: Most comfortable levels; MI: Monosyllabic identification; MXM: Neurilic digisonic; NRI: Neural response imaging; NRT: Neural response telemetry; NRT-T: NRT threshold; NucleusCI24I(CS): Nucleus 24 contour; OAE: Otoacoustic emissions; PP: Pitch pattern; QOL: Quality of life; SNHL: Sensorineural hearing loss; SNR: Signal to noise ratio; T levels: Thresholds; TI: Trochee identification

\section{Acknowledgements}

The authors would like to express their deep appreciation to Gamal Youssef, ass professor of phoniatrics Alexandria University for his support in reviewing data related to speech and language.

\section{Authors' contributions}

All authors contributed to the study conception and design. Material preparation, data collection, and analysis were performed by RE and AMK. The first draft of the manuscript was written by RE and all authors commented on previous versions of the manuscript.MS reviewed the scientific material and statistics. AK and AT reviewed the work from the Surgeon ENT point of view. All authors read and approved the final manuscript.

\section{Funding}

No funding

\section{Availability of data and materials}

The datasets during and/or analyzed during the current study available from the corresponding author on a reasonable request.

\section{Ethics approval and consent to participate}

Ethics committee approval obtained from Menoufia University. As this research extracted from bigger work of MD thesis. The protocol of the thesis was accepted on 2014. Informed written Consent to participate in the study was provided by all participants. All the audiological tests included in the study were doe on a routine clinical basis in our pre and post implanted patients with no matter of invasive technique concerning the privacy of our patients.

\section{Consent for publication}

Not applicable.

\section{Competing interests}

The authors declare that they have no competing interests.

\section{Author details}

${ }^{1}$ Hearing and Balance Clinic, ENT Department, Dubai Hospital,DHA, Abu Bakr Al Siddiq Street, Dubai 7272, United Arab Emirates. ${ }^{2}$ Faculty of Medicine, Zagazig University, Zagazig, Egypt. ${ }^{3}$ Faculty of Medicine, Cairo University, Cairo, Egypt. ${ }^{4}$ Military Medical Academy, Belgrade, Serbia. ${ }^{5}$ Faculty of Medicine, Menoufia University, Al Minufiyah, Egypt.
Received: 29 March 2020 Accepted: 11 June 2020

Published online: 02 September 2020

\section{References}

1. Basura GJ, Eapen R, Buchman CA (2009) Bilateral cochlear implantation: current concepts, indications, and results. Laryngoscope. 119(12):2395-2401

2. Papsin BC, Gordon KA (2008) Bilateral cochlear implants should be the standard for children with bilateral sensorineural deafness. Curr Opin Otolaryngol Head Neck Surg. 16(1):69-74

3. Sparreboom M, van Schoonhoven J, van Zanten BG, Scholten RJ, Mylanus EA, Grolman W, Maat B (2010) The effectiveness of bilateral cochlear implants for severe-profound deafness in children: a systematic review. Otol Neurotol. 31(7):1062-1071

4. Litovsky RY, Johnstone PM, Godar S, Agrawal S, Parkinson A, Peters R, Lake J (2006 Feb) Bilateral cochlear implants in children: localization acuity measured with a minimum audible angle. Ear Hear. 27(1):43-59

5. Sharma A, Nash A (2009) Brain maturation in children with cochlear implants. The ASHA Leader. 14(5):14-17

6. Zwolan TA, Implantable hearing device. In: Handbook of clinical audiology. Katz J, Chasin M, Hood J, et al; (Eds.) 7th edition. Copyright (c) Wolters Kluwer Health. 2015;p.817-849

7. Peters BR, Wyss J, Manrique M (2010) Worldwide trends in bilateral cochlear implantation. Laryngoscope. 120(2):S17-S44

8. Abdel Maksoud A, Kamal N, Soliman S (1990) Development of and administration of selected items of Arabic Minimal Auditory Capabilities (MAC) test: continuation study. Faculty of Medicine, Ain Shams University, M.D. Thesis

9. El-Kholi W (2000) Development of an Arabic pediatric speech perception test battery. The Egyptian Journal of Otolaryngology. 18(2):23

10. Wilson BS, Dorman MF (2008) Cochlear implants: current designs and future possibilities. J Rehabil Res Dev. 45(5):695-730

11. Ramsden $R$, Greenham P, O'Driscoll M, Mawman D, Proops D, Craddock L, Fielden C, Graham J, Meerton L, Verschuur C, Toner J, McAnallen C, Osborne J, Doran M, Gray R, Reiss LA, Fowler JR, Hartling CL, Oh Y (2018) Binaural pitch fusion in bilateral cochlear implant users. Ear Hear. 39(2):390-397

12. Litovsky RY, Parkinson A, Arcaroli J (2009) Spatial hearing and speech intelligibility in bilateral cochlear implant users. Ear Hear. 30(4):419-431

13. Verschuur CA, Lutman ME, Ramsden R, Greenham P, O'Driscoll M (2005 Sep) Auditory localization abilities in bilateral cochlear implant recipients. Otol Neurotol. 26(5):965-971

14. Kerber S, Seeber BU (2012) Sound localization in noise by normal-hearing listeners and cochlear implant users. Ear Hear. 33(4):445-457

15. Gordon KA, Wong DD, Papsin BC (2010 Oct) Cortical function in children receiving bilateral cochlear implants simultaneously or after a period of interimplant delay. Otol Neurotol. 31(8):1293-1299

16. Key AP, Porter HL, Bradham T (2010) Auditory processing following sequential bilateral cochlear implantation: a pediatric case study using event related potentials. J Am Acad Audiol. 21(4):225-238

17. Baudonck N, Dhooge I, D'haeseleer E, Van Lierde K (2010) A comparison of the consonant production between Dutch children using cochlear implants and children using hearing aids. International journal of pediatric otorhinolaryngology. 74:416-421

18. Fernandes NF, Morettin M, Yamaguti EH, Costa OA, Bevilacqua MC (2015) Performance of hearing skills in children with auditory neuropathy spectrum disorder using cochlear implant: a systematic review. Braz J Otorhinolaryngol 81:85-96

19. Chadha NK, Papsin BC, Jiwani S, Gordon KA (2011) Speech detection in noise and spatial unmasking in children with simultaneous versus sequential bilateral cochlear implants. Otol Neurotol. 32(7):1057-1064

20. Reiss LAJ, Fowler JR, Hartling CL, Oh Y (2018) Binaural pitch fusion in bilateral cochlear implant users. Ear Hear. 39(2):390-397

\section{Publisher's Note}

Springer Nature remains neutral with regard to jurisdictional claims in published maps and institutional affiliations. 